\title{
Apport de la modélisation et des populations virtuelles pour transposer les résultats des essais cliniques à la vie réelle et éclairer la décision publique
}

\author{
François Gueyffier ${ }^{1}$, Catherine Brun Strang ${ }^{2}$, Gilles Berdeaux ${ }^{3}$, Lionel Riou França ${ }^{4}$, Patrick Blin ${ }^{5}$, \\ Jacques Benichou ${ }^{6}$, Jacques Massol $^{7}$ et les participants à la table ronde $N^{\circ} 6$ de Giens XXVII*
}

1 Service de Pharmacologie Clinique et essais thérapeutiques, Hospices Civils de Lyon, France \& UMR5558, CNRS et Université Lyon 1 , Lyon, France

2 Global Medical Affairs, Sanofi R\&D, Chilly-Mazarin, France

3 Conservatoire National des Arts et Métiers, Paris, France

4 PhiSquare, Paris, France

5 Service de Pharmacologie CIC-P 0005, Université Bordeaux Segalen, Bordeaux, France

6 Unité de Biostatistiques, Université de Rouen, Rouen, France

7 PhiSquare Fondation Transplantation Paris ; Centre Hospitalo Universitaire de Besançon, Hôpital Saint Jacques, Besançon, France

Texte reçu le 20 mars 2012 ; accepté le 4 juin 2012

\section{Mots clés :}

modélisation simulation ; essais cliniques ; décision publique ; populations virtuelles

\begin{abstract}
Résumé - La modélisation permet d'agréger un ensemble de connaissances, selon une structure et des règles déterminées. Elle permet l'étude du comportement de systèmes plus ou moins complexes par des simulations. La modélisation est utilisée dans différents domaines technologiques de pointe (météorologie, aéronautique). Elle devient reconnue pour l'évaluation thérapeutique des médicaments et dispositifs médicaux, de leur conception à la prescription (autorisation de mise sur le marché [AMM], remboursement, fixation des prix et réinscriptions). Elle suit une démarche scientifique et fait l'objet de recommandations de bonnes pratiques. Le couplage de modèles à des populations virtuelles permet de rendre des résultats réalistes à l'échelle de populations, et de tester des stratégies diagnostiques ou thérapeutiques, ainsi que les conséquences liées à la transposition des résultats d'essais cliniques. À l'aide d'exemples, les participants de la table ronde ont analysé les apports du couplage de la modélisation aux populations virtuelles et proposent des recommandations pour leur utilisation judicieuse systématique.
\end{abstract}

Abréviations : voir en fin d'article.

\section{Introduction}

Nous modélisons tous et depuis longtemps. Lorsque Magritte peint une pipe et provoque le spectateur de son tableau en précisant : «Ceci n'est pas une pipe », il nous invite à nous rappeler le décalage permanent entre la réalité et ce que nous en percevons. Nos perceptions sont organisées en modèles, plus ou moins parcellaires, limités ou complexes, nous offrant des outils support d'échanges (le langage, oral ou écrit, est un modèle, ou un ensemble de modèles), permettant de nous comprendre mutuellement, et surtout de prévoir le résultat de nos actions. La modélisation par le langage mathématique présente des avantages remarquables de puissance et d'efficacité, rendus particulièrement accessibles avec les développements technologiques informatiques. La construction de modèles mathématiques peut prétendre aujourd'hui à des niveaux de complexité dépassant de très loin la capacité d'abstraction et de prédiction du cerveau humain, et prolongeant de fait ses rêves de développement.

Une des premières modélisations en santé a été formalisée dès 1760 par Bernoulli dans un mémoire de l'Académie des sciences de Paris, ${ }^{[1]}$ où il proposait la modélisation d'une épidémie de variole, afin de prévoir le rapport bénéfice-risque de l'inoculation de la maladie dans une population sujette à cette épidémie. La modélisation formelle est largement utilisée dans d'autres

* Pour la liste des participants, voir en fin d'article. 
domaines que la santé, comme l'environnement avec la météorologie, ou l'aéronautique pour la construction de prototypes.

La question de la transposition des résultats des essais cliniques se pose à toutes les étapes de la vie administrative du médicament : lors de l'estimation de la balance bénéfice-risque pour l'enregistrement, lors de la quantification de l'intérêt thérapeutique pour l'admission au remboursement, lors de la négociation des prix et des volumes pour le contrat avec le Comité économique des produits de santé (CEPS), lors de la réinscription. Les réponses à ces diverses questions peuvent reposer sur des avis d'experts, une règle de proportionnalité définie sur des hypothèses simplissimes, ou sur l'élaboration d'un modèle intégrant des hypothèses plus complexes.

Les décisions sur le médicament (qu'il s'agisse de l'autorisation de mise sur le marché [AMM] ou des décisions d'accès au remboursement) se fondent essentiellement sur les données des essais dont on sait qu'ils ne représentent pas strictement ce qui va se passer dans la vie réelle. Les nombreuses incertitudes qui entourent les décisions publiques concernent non seulement les aspects biologiques avec leur variabilité, les décisions cliniques mais aussi la transposabilité des données des essais à la vraie vie. Pour que les décideurs puissent prédire ou quantifier de manière optimale le bénéfice attendu du médicament il apparaît nécessaire de recourir à des modèles dont la complexité permet d'approcher suffisamment la réalité, afin de prendre les décisions publiques de la façon la plus éclairée.

Devant ce constat, les participants de la table ronde 6 se sont fixés pour objectif d'évaluer, sur la base d'exemples concrets, le rôle de la modélisation en santé et ses conditions d'acceptabilité pour transposer les résultats des essais cliniques et éclairer la décision publique.

\section{Quelques définitions préliminaires}

- Transposition : application des données (résultats d'essais cliniques randomisés et de méta-analyses par exemple) à des populations et à des conditions différentes de celle(s) sur lesquelles elles ont été recueillies. ${ }^{[2]}$ N.B. : les décisions médicales représentent toujours l'extrapolation de données recueillies dans le passé sur d'autres individus, à une nouvelle situation, afin d'infléchir au mieux (ou de respecter...) ce que l'on prédit de l'évolution de sa situation.

- Population virtuelle réaliste : population simulée informatiquement sur la base de règles de connaissance existantes (épidémiologiques et environnementales) pour reconstituer in silico une population réelle et son évolution. Les populations virtuelles sont des outils intéressants lorsque le rapport bénéficerisque populationnel, qui est l'agrégation des rapports bénéfice-risque individuels, n'est pas directement calculable sur un profil individuel représentatif en raison de la complexité des règles associatives et du caractère multidimensionnel des fonctions en jeu. La population virtuelle peut naturellement devenir le support d'exploration de différents scénarios d'utilisation d'un médicament ou d'une stratégie thérapeutique plus ou moins complexe. En fonction de contraintes extérieures, ces analyses stratégiques peuvent conduire à l'identification de seuils de bénéfice, référentiel d'indication thérapeutique rationnelle. La genèse d'une population virtuelle doit viser au plus grand réalisme possible, par sa représentativité et la capture des traits les plus essentiels de la population réelle : c'est le concept de population virtuelle réaliste (PVR).

- Modèle : représentation simplifiée d'un processus complexe de la réalité. Exemple : le modèle statistique représente le processus de survenue de résultats expérimentaux et estime la probabilité d'observer ces résultats sous l'hypothèse nulle, c'est-àdire l'hypothèse d'égalité entre deux grandeurs comparées. Le calcul des effectifs nécessaires à une étude fait intervenir un modèle formulant deux hypothèses, nulle et alternative, et incluant une estimation a priori de la taille de la différence cliniquement pertinente, la variabilité des mesures des grandeurs, et les risques d'erreur positive (alpha) ou négative (bêta).

L'élaboration de modèles mathématiques est une démarche pluridisciplinaire. Un des critères de qualité importants de cette démarche est sa reproductibilité, acquise grâce à la transparence des étapes de cette élaboration. Les modèles produits sont actualisables, et peuvent être récupérés dans leur globalité ou pour partie dans d'autres projets visant d'autres objectifs. Appliquée au domaine thérapeutique, cette démarche permet de quantifier les effets attendus, bénéfiques ou indésirables, mais aussi l'incertitude associée aux résultats qu'elle fournit en estimant les risques d'erreur aux différentes étapes. Elle présente tout son intérêt dans des situations complexes. La modélisation est un outil classique pour traiter les données de pharmacocinétique et pharmacodynamique.

- Approche populationnelle : le recours aux approches populationnelles en modélisation pharmacologique permet de prendre en compte les parts de la variabilité expliquées et non expliquées dans la construction des modèles. En pratique, la construction de modèles paramétrés sur des échantillons représentatifs ou non, puis couplés à des populations virtuelles, réalistes ou non, présente un intérêt à toutes les étapes de développement, en amont et au-delà de la phase administrative d'admission au marché/remboursement. La modélisation est l'unique façon de prédire, en général, par la synthèse formalisée et opératoire des informations disponibles à un moment donné, qu'il s'agisse des résultats d'une étude de phase I, II ou III, ou des données post AMM.

\section{Partage d'expérience}

De nombreuses modélisations sont réalisées en santé et les contextes et les méthodes en sont variés. À partir d'exemples et de situations brièvement exposés ci-dessous, les participants de la 
table ronde 6 ont échangé expériences et réflexions pour aboutir à des propositions et recommandations concernant l'utilisation de l'approche modélisation dans les données d'accès au marché, focalisée mais non exclusivement, sur les questions de transposition.

\section{1. Une modélisation déjà bien ancrée dans les pratiques : exemple des vaccins}

Dans le cadre de l'audition annuelle des firmes pharmaceutiques par le Comité technique des vaccinations (CTV) ${ }^{[3]}$ des données complémentaires par rapport à l'AMM peuvent être apportées y compris des données de modélisation de l'impact des vaccins en France. Si le dossier est complexe, un partenariat avec l'Institut de veille sanitaire (InVS) est systématiquement recommandé pour réaliser une modélisation de l'impact du vaccin en termes de morbi-mortalité ou en termes médico-économiques.

\subsection{Utilisation des populations virtuelles pour mesurer l'impact d'un nouveau traitement dans le choc septique}

L'impact de l'utilisation de la drotrécogine alpha dans le choc septique a été mis en question la suite de la publication des résultats de l'étude PROWESS, ${ }^{[4]}$ montrant un gain sur la mortalité dans le choc septique. Outre celles de l'étude PROWESS, les données utilisées pour constituer le modèle prédictif de l'impact du médicament étaient issues d'un réseau de services de réanimation français CUB-Réa, ${ }^{[5]}$ renseignant la distribution des caractéristiques de patients pris en charge dans ces services : type de séjour, sévérité, durée de séjour, défaillances, etc. Ces données ont permis de construire des sous-groupes de patients, d'autres informations issues de la littérature permettant de redresser ces distributions lorsque certaines strates de patients étaient sur ou sous-représentées dans CUB-Réa : ainsi, la population virtuelle issue de la base CUB-Réa est devenue plus réaliste, en ce sens qu'elle représentait mieux les données réelles de la population des services de réanimation français, permettant d'estimer un effet thérapeutique attendu moyen. ${ }^{[6]}$

\subsection{Population virtuelle et modélisation pour simuler un impact long terme : exemple du glaucome}

Le glaucome demeure dans les pays développés une des causes majeures de cécité. Les patients dont la pression intraoculaire est élevée ont un risque plus important de développer la maladie ou que celle-ci progresse. Il a été démontré que le contrôle de la pression intraoculaire avait un impact sur l'apparition du glaucome ou sur son évolution. Le contrôle de la pression intraoculaire est réalisé à l'aide de moyens thérapeutiques (collyres) ou physiques (chirurgie, laser). La décision d'accès au marché des traitements du glaucome repose principalement sur le contrôle de la pression intraoculaire. Une étude de morbidité sur la cécité nécessite un suivi de plusieurs années, difficilement compatible économiquement avec la durée consentie de monopole liée au brevet. L'utilisation de modèle devient donc nécessaire pour prédire le bénéfice sur la morbidité à partir des résultats sur la pression.

Afin de recueillir les données nécessaires à paramétrer le modèle, une première enquête a été conduite en France, sur un échantillon d'ophtalmologistes tirés au sort. Elle a quantifié les facteurs de risques de la progression du glaucome et ceux associés à des coûts élevés, en situation de prescription. Les effets indésirables et les changements de traitement étaient associés avec une plus grande probabilité d'avoir une progression du glaucome. ${ }^{[7]}$ Les changements de traitements et la progression de la maladie contribuaient à des coûts plus élevés. ${ }^{[8]}$ Une chaine de Markov a été construite afin d'agréger les fonctions de risques décrites cidessus. ${ }^{[9,10]}$ L'horizon temporel du modèle était la vie entière. Les probabilités de décès ont été modélisées à partir des tables françaises de mortalité. Les patients pouvaient bénéficier de 4 lignes de traitement sous forme de collyres, et avaient ensuite accès à la chirurgie ou au laser; les non répondeurs recommençaient les lignes de collyres, ceci jusqu'au décès. Les probabilités d'échec thérapeutiques provenaient soit de bases de données de prescription (par exemple, la general practice research database en Grande Bretagne [UK GPRD]) soit d'essais cliniques. Les résultats de ce modèle ont été validés par confrontation à des données réelles. Le modèle a donc été montré ainsi capable de reproduire les résultats d'essais de morbidité de long terme (par exemple, l'ocular hypertension treatment study).

Cette approche, relativement classique en économie de la santé, permet d'agréger des données provenant de sources diverses, d'essais thérapeutiques et de données de vie réelle, autorisant ainsi de nombreuses extrapolations utiles lors d'une primo inscription et qui pourraient être ainsi vérifiées lors d'une réinscription. Le nombre de règles de connaissance, la complexité des règles d'association et les sources nombreuses d'incertitudes ont rendu obligatoires l'approche stochastique et la génération de population virtuelle.

\subsection{Population virtuelle et simulation de stratégies préventives : exemple de l'hypertension artérielle}

Marchant et al. ont généré une population virtuelle réaliste française entre 35 et 64 ans, reproduisant la distribution des facteurs de risque classiques, permettant ainsi de calculer le risque cardiovasculaire pour chaque individu de la population constituée. Les données source étaient empruntées à l'enquête monitoring of trends and determinants of cardiovascular diseases (MONICA) ${ }^{[11]}$ France, la distribution en âge et sexe des individus était calquée sur 
les statistiques nationales de l'Institut national de la statistique et des études économiques (INSEE). ${ }^{[12]}$ La validation externe a comporté notamment la confrontation des scores de risque prédits moyens par classes d'âge pour les femmes et les hommes aux statistiques de mortalité nationale, en utilisant deux scores de risque cardiovasculaire reconnus par un groupe de travail ad hoc de l'Agence nationale d'accréditation et d'évaluation en santé (ANAES), SCORE et Framingham. Cette étape de validation a permis d'illustrer que les équations de risque de Framingham présentaient entre elles des incohérences avec une mortalité coronarienne prédite supérieure à la mortalité cardiovasculaire, chez les hommes d'âge moyen. L'analyse de cette incohérence, permise par la disponibilité des données de chaque individu de la population virtuelle, a mis en évidence des coefficients trop importants donnés pour la mortalité coronarienne au tabac et au rapport cholestérol total/HDL. ${ }^{[13]}$ Cette population virtuelle a été le support d'une comparaison entre l'application théorique des recommandations sur le traitement médicamenteux antihypertenseur, ${ }^{[14]}$ et les résultats d'une enquête épidémiologique représentative. ${ }^{[15]}$ Cette analyse a montré que, si les recommandations ne semblent pas mises en pratique concernant le dépistage de l'hypertension artérielle, elles ne sont pas non plus bien suivies pour la prise en compte du risque puisque les jeunes hypertendues étaient traitées bien au-delà de ce que leur bas niveau de risque aurait justifié. Il a aussi été très simple de montrer que le changement de seuil de définition de l'hypertension artérielle, de 160/95 à 140/90 mm Hg, avait simplement doublé la prévalence de l'hypertension artérielle en France entre 35 et 64 ans. Cela illustre la capacité directe que ces populations virtuelles réalistes offrent d'estimer la taille des populations cibles d'une thérapeutique.

La sélection des individus à traiter sur leur niveau de risque prédit, prônée sans succès depuis plus de 30 ans par les recommandations, est mathématiquement la plus efficace, ${ }^{[16]}$ si l'on en reste au nombre d'événements évités. Cependant, elle donne le même poids aux accidents qui surviennent à 30 ou à 80 ans. La même population virtuelle a permis de simuler ce que devraient être les seuils de risque prédit pour l'instauration du traitement antihypertenseur si l'on souhaitait réduire les inégalités entre sexes et surtout entre générations, induites par l'application directe de la sélection des individus sur leur niveau de risque. L'objectif innovant de la nouvelle stratégie a été défini comme la prévention de la même proportion d'accidents attendus pour toutes les tranches d'âge, pour les femmes comme pour les hommes. Plus égalitaire, la nouvelle stratégie est plus coûteuse pour un même nombre d'accidents prévenus, mais les accidents prévenus surviennent chez des individus plus jeunes: la nouvelle stratégie prévient donc plus d'années de vie en bonne santé. Ce dernier exemple illustre l'intérêt de l'outil population virtuelle réaliste pour mettre au point des stratégies répondant à des objectifs médico-économiques complexes car multidimensionnels : prise en compte du niveau de pression artérielle, du risque prédit, de la distribution des risques prédits par âge et par sexe, de l'espérance de vie des individus. ${ }^{[17]}$

\subsection{Modélisation de l'impact de santé publique d'un antidiabétique sur plusieurs critères}

Les discrete event simulation (DES) sont fréquemment utilisés dans le domaine de l'ingénierie pour simuler des systèmes complexes et commencent à l'être dans le domaine médical (46 publications dans Pubmed au 17 mars 2012). Une DES est une technique de simulation qui estime séquentiellement à chaque temps $t$, sur une période de temps défini, le devenir de chaque individu (entité) d'une population virtuelle réelle en terme d'événements (exemple : infarctus, récidive d'infarctus, complication micro-vasculaire, décès) en fonction de ses caractéristiques initiales et de leur évolution au cours du temps (facteurs de risque), ainsi que des interventions au cours du temps (exemple : traitements, effets indésirables, switches, arrêts de traitement, hospitalisations) et des événements précédents. ${ }^{[18,19]}$ L'un des principaux avantage des DES est de pouvoir utiliser simultanément plusieurs fonctions ou modèles mathématiques, spécifiques de chaque événement étudié, pour modéliser des situations complexes et compétitives. ${ }^{[20,21]}$

JJ Caro a utilisé l'approche DES pour estimer l'intérêt de Santé publique du rimonabant dans une population française diabétique obèse ou en surpoids. ${ }^{[18]}$ Les caractéristiques de la population virtuelle étaient issues des données démographiques françaises, des études MONICA, l'étude Échantillon national témoin représentatif des personnes diabétiques (ENTRED) et l'étude prise en charge du diabète de type 2 en France (étude ECODIA). L'évolution des facteurs de risque et des interventions au cours du temps était estimée à partir d'équations prédictives selon les caractéristiques du patient et l'effet du traitement observé dans l'étude rimonabant in obesity and related disorders (RIO Diabetes). ${ }^{[22]}$ Les fonctions de risque utilisées étaient l'équation de Framingham, corrigée pour la France pour le premier événement cardiovasculaire, les données de la base de données du Saskatchewan pour les récidives d'événements cardiovasculaires, les données de l'étude United Kingdom prospective diabetes study (UKPDS) pour les complications micro-vasculaires et les données de mortalité française pour les décès.

\section{Discussion}

Un grand nombre de questions de santé publique ne reçoivent de réponses qu'à partir de l'utilisation de modèles mathématiques, validés lorsque l'exercice est possible par confrontation des prédictions avec les observations recueillies souvent à partir de longues séries constituées dans le temps et l'espace.

Dans le contexte d'une situation de prévention (mortalité par des médicaments hypocholestérolémiant, cécité par les anti-glaucomateux, etc.), les essais cliniques randomisés fournissent une estimation du bénéfice relatif en moyenne, directement sur le critère clinique d'intérêt, ou sur un critère intermédiaire comme la cholestérolémie ou la pression intraoculaire. Si la démonstration du bénéfice n'est apportée que sur le critère intermédiaire, l'enregistrement éventuel 
fait l'hypothèse que le critère intermédiaire réunit les propriétés d'un bon critère de substitution. ${ }^{[23]}$ Il n'était pas dans l'objectif de la table ronde de discuter cette problématique, mais il est de plus en plus reconnu qu'il n'existe pas de critère de substitution parfait. ${ }^{[24]}$ On peut imaginer facilement deux limites au modèle du critère de substitution : 1) les mécanismes en jeu sont souvent multiples et les effets cliniques ne peuvent pas être expliqués dans leur ensemble par l'action sur le critère de substitution ; le modèle de prédiction devrait alors prendre en compte plusieurs critères de substitution partiels pour être plus efficace ; 2) les effets indésirables obéissent le plus souvent à d'autres mécanismes que le mécanisme représenté par le critère de substitution. La modélisation de l'impact de santé publique requiert que la relation entre critère intermédiaire et critère clinique soit univoque et claire, ce qui n'est en général pas accessible à une démonstration. Cette relation peut être testée par exemple en méta-régression, mais un tel test présente des biais potentiels importants, notamment le biais écologique. Dans la suite de l'exemple, on considère le cas plus satisfaisant d'une démonstration de l'effet directement sur le critère clinique. La population étudiée dans les essais cliniques randomisés est facilement identifiable grâce aux critères d'éligibilité. Lorsque aucun modificateur du bénéfice relatif n'a été mis en évidence, cette estimation est donc jusqu'à preuve du contraire extrapolable à tout patient traité : le niveau de bénéfice est calculable pour tous les individus de la population traitée. Dans ces conditions, la modélisation est simple, pourvu i) qu'un score de risque soit disponible ; et ii) que la distribution des facteurs de risque dans la population permette de définir la structure et la taille de la population cible. L'application du score de risque et du bénéfice relatif permet de calculer le nombre d'accidents prévenus. La même procédure s'applique pour les effets indésirables.

La modélisation suit le cahier des charges complet de toute démarche scientifique, avec définition préalable des objectifs du travail, l'exploration de la validité interne du modèle par la définition précise des hypothèses sous-jacentes, l'exploration de leur niveau de fiabilité (cf. l' analyse du niveau de preuve des essais pris en compte), l'exposé transparent des méthodes employées. Les paramètres des équations du modèle peuvent être issus de la littérature, ou optimisés pour réduire la distance entre les prédictions du modèle et des données externes.

La validité externe du modèle nécessite la confrontation des prédictions à des observations différentes de celles ayant servi à la valorisation des paramètres. L'élaboration de modèles médicoéconomiques a fait l'objet de recommandations notamment par l'International Society for pharmacoeconomics and outcomes research (ISPOR) ${ }^{[25]}$ ou localement par le Collège des économistes de la santé français.

Les limites de cette technologie doivent être bien comprises et analysées afin de donner à la démarche toute sa place. Les limites techniques concernent notamment la disponibilité des données source et leur fiabilité, en parallèle au niveau de preuve des hypothèses sous-jacentes. Ces limites pourront être reculées notamment

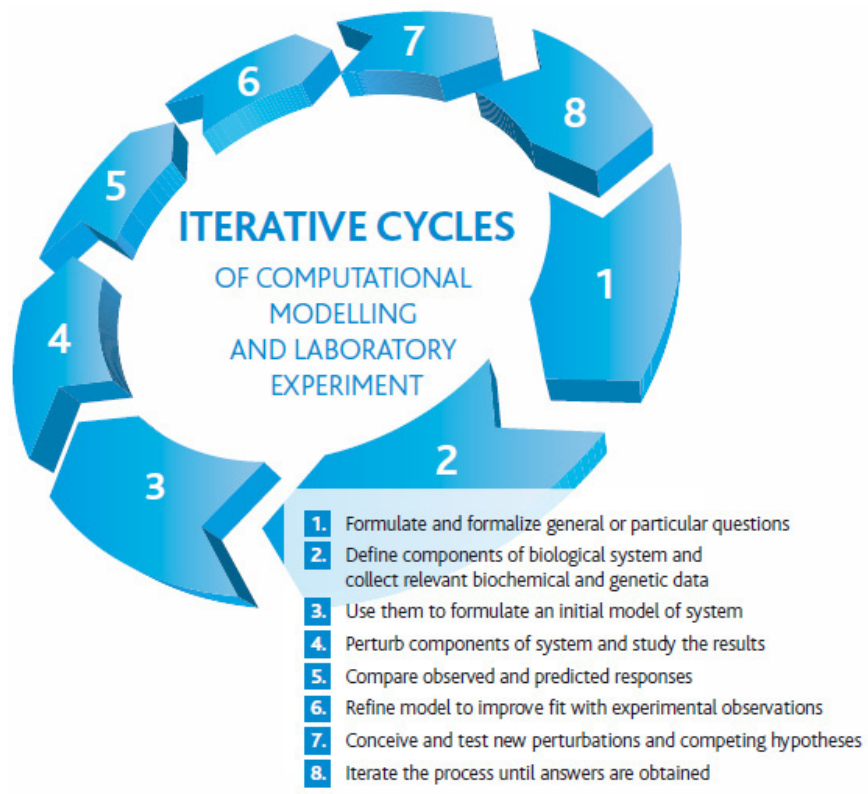

Fig. 1. Le cyle de construction des modèles en interaction avec les expériences et les observations extérieures au modèle. ${ }^{[26]}$ Avec l'aimable autorisation de Charles Auffray (communication personnelle). Accessible sur http:// www.eisbm.org/include/pdf.eisbm_corpratebrochure.pdf

lorsque les études épidémiologiques et les essais cliniques seront conduits en suivant une logique d'intégration dans les modèles, tenant compte de l'existant et des questions essentielles pour l'amélioration de leurs performances. Le développement d'interactions entre la construction des modèles et la conduite des expériences biologiques et cliniques est essentiel, et contribue à établir le cercle vertueux de l'intégration des connaissances (figure ${ }^{1}$ ). ${ }^{\text {[26] }}$

Il faut souligner aussi que les modèles actuels sont essentiellement des modèles phénoménologiques, qui décrivent par des fonctions mathématiques la surface des phénomènes observés et non leurs mécanismes intimes. La prise en compte de ce niveau de complexité doit faire intégrer la modélisation en pharmacologie classique (pharmacocinétique/pharmacodynamie ou PK/PD) avec les approches de biologie et de physiopathologie systémique : physiologically based pharmacokinetic (PBPK) et physiologically based pharmacodynamic (PBPD).

Au-delà des limites purement techniques, la démarche est confrontée à des obstacles psychologiques : la carence culturelle des différents acteurs dans le développement et la régulation du médicament explique l'impression de manque de transparence qu'ils ressentent devant un résultat de modélisation, comme s'ils étaient confrontés à une boîte noire, rendant difficile le partage réel des résultats. De plus, il faut que les modèles prédictifs fassent l'objet d'une vérification en vie réelle. L'une des meilleures façons de faire accepter les modèles est de montrer par des études en vie réelle que ce qui est observé correspond, à une marge d'erreur près, à ce qui était prédit. 
S'y ajoutent les problèmes de conflit d'intérêt, non spécifiques aux modèles, mais ne les épargnant pas : ces problèmes incluent la compétition entre modélisateurs et experts - key opinion leaders non modélisateurs, ou l'orientation non scientifique du travail de modélisation guidée par des intérêts financiers ou promotionnels. Dans ce domaine aussi, il est important que les acteurs gardent une réelle indépendance vis-à-vis des intérêts divers en jeu, ne gardant comme finalité réelle que l'intérêt de santé publique. Aussi les modélisations doivent être effectuées de façon transparente et reproductible avec un comité scientifique qualifié et indépendant, à l'instar des études demandées par les autorités.

Les autorités de remboursement telles le National Institute for Health and Clinical Excellence (NICE) par exemple peuvent requérir la modélisation pour synthétiser les données disponibles et permettre de fournir une estimation de l'efficacité clinique et du rapport coût-efficacité ${ }^{[27]}$ lorsque : toutes les données ne proviennent pas du même essai clinique ; les patients participant à l'essai n'ont pas les mêmes caractéristiques que les patients destinés à bénéficier du traitement au sein du NHS ; les critères de résultat utilisés ne sont ni le Health Related Quality of Life ni la survie ; aucun comparateur n'a été utilisé ; les sous-groupes n'ont pas été définis ; les bénéfices à long terme ne peuvent pas être mesurés dans la période de suivi. Or les recommandations du NICE influencent les processus décisionnels d'autres pays d'Europe, des ÉtatsUnis et de pays émergents. L'absence d'avis favorable du NICE pour un médicament est perçue de nos jours comme une barrière importante à son succès sur les autres marchés.

En renforçant les compétences en modélisation en France, en disposant de plateformes épidémiologiques nationales et mondiales on disposera d'une recherche capable de traiter les questions que seuls les modèles mathématiques peuvent espérer aborder en santé publique.

\section{Recommandations}

\subsection{Pour les acteurs}

La réussite d'une démarche de transposition par modélisation sur population virtuelle nécessite un interfaçage efficace de tous les acteurs clés, qui doivent être associés notamment dans l'encadrement de la démarche à travers des interactions bien formalisées. Les différentes entités concernées doivent être représentées dans cet encadrement : AMM, Commission de la transparence (CT), Haute autorité de santé (HAS), CEPS, INVS, industriels, académiques... Cet effort d'encadrement doit être réalisé en lien avec les groupes de travail déjà existants tant nationaux qu'internationaux: experts de l'International Society of Pharmacoeconomics \& Outcomes Research (ISPOR), groupe d'harmonisation des méthodes d'estimation des rapports bénéfice-risque (RBR) dans les dossiers soumis à l'Agence européenne du médicament (EMA), le groupe Eunet HTA qui vise à harmoniser les méthodes d'évaluation en vue du remboursement...

Les attendus d'un tel encadrement sont d'aboutir à des conseils précis aux industriels lorsqu'ils sollicitent la HAS, la CT ou le CEPS sur l'utilisation d'un modèle dans leurs dossiers.

Les différents acteurs, qu'ils soient régulateurs, industriels ou experts académiques, utilisateurs ou décideurs, doivent être sensibilisés, et mieux formés aux concepts sous-jacents à la démarche et à ses limites, mais aussi au caractère indispensable de leur utilisation dans le cadre d'une aide à la décision éclairée. La prégnance attendue de la démarche, de ces concepts, rend nécessaire la formation des médecins utilisateurs finaux, que ce soit en formation médicale initiale ou continue. Dans le même esprit, le grand public doit être familiarisé avec ces nouveaux outils et ce qu'ils peuvent apporter.

\subsection{Pour la recherche}

L'ampleur des développements nécessaires en modélisation autour du développement du médicament et de la construction des populations virtuelles justifie le fléchage de financements comme l'inscription d'une ligne spécifique au programme hospitalier de recherche clinique (PHRC), aux programmes de l'Agence nationale de la recherche (ANR), ou dans le cadre du programme cadre de recherche et de développement (PCRD) de l'Union européenne. Il paraît nécessaire d'encourager le montage de structures de modélisation, comme l'European Institute For Systems Biology and Medicine. ${ }^{[28]}$

La prise en compte de l'importance de l'approche et du développement des outils nécessaires devrait orienter une partie des recherches épidémiologiques vers le recueil d'informations indispensables à la construction de populations virtuelles locales ou régionales, et favoriser la mise en commun des informations essentielles au sein de bases de données individuelles des essais cliniques et des études épidémiologiques.

\section{Conclusion et recommandations des membres de la table ronde}

Les participants de la $6^{\mathrm{e}}$ table ronde de Giens 2011 recommandent : (i) que le recours à la modélisation soit systématiquement envisagé et, s'il est décidé, que la modélisation soit conduite, interprétée, validée et appliquée de façon adaptée ; (ii) dans la mesure où la modélisation et la simulation sont par nature des approches multidisciplinaires, que toutes les compétences professionnelles requises soient mises en œuvre, à tous les stades ; (iii) que toutes les données requises pour une modélisation optimale soient accessibles, grâce à un partenariat approprié entre industriels, universitaires, régulateurs et autorités de santé ; (iv) que les cliniciens soient 
systématiquement conscients de ses apports potentiels, et qu'ils aient l'expérience au moins de l'interprétation de ses résultats ; (v) que les spécialistes du domaine développent des algorithmes ou arbres de décision pour aider les non-spécialistes à décider quand il est nécessaire de recourir à la modélisation ; (vi) que les universités proposent aux professionnels du médicament des formations appropriées dans le domaine de la modélisation et de la simulation. ${ }^{\text {[29] }}$

Plusieurs priorités peuvent ainsi être dégagées qui devraient être prises en compte dans les politiques de recherche :

1. Développement méthodologique : la description des variabilités contextuelles, en elle-même, et l'analyse de ces déterminants nécessite des développements méthodologiques importants.

2. Interdisciplinarité : le soutien à des équipes et des réseaux d'équipes réunissant des disciplines différentes (sociologie, épidémiologie, santé publique, statistiques, informatiques et mathématiques) constitue aussi une priorité. De tels réseaux devraient être, non seulement soutenus par des interventions spécifiques, mais aussi particulièrement valorisés dans les différentes institutions de recherche elles-mêmes, y compris en prenant en compte les modalités d'évaluation scientifique, souvent différentes entre les dites disciplines. L'implication individuelle dans l'interdisciplinarité devrait être perçue comme un aboutissement et reconnue à la hauteur des enjeux, au lieu d'être vécue comme un parcours d'obstacle sans espoir de reconnaissance.

3. Constitution de bases de données : un des obstacles majeurs au développement des travaux d'analyse contextuelle réside dans la rareté des données individuelles (et non pas agrégées), géoréférencées (au niveau le plus fin possible, dans tous les cas infracommunal), sociales et sanitaires. Si elle n'est pas un travail de recherche en soi, la constitution de telles bases de données (par le rapprochement de données issues des différents systèmes d'information existants : recensement, registres de maladies, données de l'Assurance maladie, données de mortalité, maladies à déclaration obligatoire, cohortes développées dans les EPST, données de l'Institut de recherche et documentation en économie de la santé (IRDES), de l'Institut national de prévention et d'éducation pour la santé (INPES, etc.) est en quelques sortes l'équivalent des « grands équipements » pour la recherche fondamentale : c'est une condition nécessaire pour développer des recherches de qualité, elle nécessite des savoir-faire spécifiques et donc des financements spécifiques, elle doit pouvoir bénéficier au plus grand nombre de chercheurs et donc s'accompagner d'un effort de transparence et d'accessibilité - évidemment accompagné de conditions de confidentialité strictes - des différentes institutions propriétaires.

\section{Groupe de travail pour le suivi de la table ronde $\mathrm{N}^{\circ} 6$}

Enfin, les participants réunis au sein de la table ronde suggèrent la mise en place d'un groupe de travail qui déclinera en actions ces recommandations. Le cahier des charges de ce groupe de travail comporte i) la création d'un lexique ou glossaire, permettant aux différents acteurs venus de différentes disciplines de s'accorder sur un langage et des concepts au contenu commun ; ii) l'élaboration de recommandations techniques pour la mise en œuvre des travaux de modélisation, l'évaluation de leur qualité et de leur validité, leur intégration dans le processus de développement et d'accès au marché ; iii) le suivi des recommandations de la TR6 Giens XXVII.

\section{Participants}

Béatrice Augendre Ferrante (Laboratoire Lilly France), Jacques Benichou (CHU, Rouen), Gilles Berdeaux (Alcon Labs), Patrick Blin (Université de Bordeaux) ; Thomas Borel (Sanofi-France), Cecile Rey-Coquais (Laboratoire Pfizer France), Jean-Michel Joubert (UCB Pharma SA), François Meyer (Haute autorité de santé), Sophie Muller (Labaratoire GlaxoSmithKline France), Leslie Pibouleau (Haute autorité de santé), Marion Pinet (Haute autorité de santé), Christel Vidal (CHU, Besançon).

\section{Conflits d'intérêts. Aucun.}

Abréviations. AMM : autorisation de mise sur le marché ; ANAES : Agence nationale d'accréditation et d'évaluation en santé ; ANR : Agence nationale de la recherche ; CEPS : Comité économique des produits de santé ; CT : Commission de la transparence; CTV : Comité technique des vaccinations ; DES : discrete event simulation; EMA : Agence européenne du médicament ; ENTRED : échantillon national témoin représentatif des personnes diabétiques ; Étude ECODIA : étude prise en charge du diabète de type 2 en France; HAS : Haute autorité de santé; INPES : Institut national d'éducation pour la santé ; INSEE : Institut national de la statistique et des études économiques; IRDES : Institut de recherche et de documentation en économie de la santé ; ISPOR : International Society for pharmacoeconomics and outcomes research; MONICA : monitoring of trends and determinants of cardiovascular diseases ; PBPD : physiologically based pharmacodynamic ; PCRD : programme cadre de recherche et de développement de l'Union européenne; NICE: United Kingdom National Institute for Health and Clinical Excellence; PBPK : physiologically based pharmacokinetic; PHRC : programme hospitalier de recherche clinique ; PK/PD : pharmacocinétique/pharmacodynamie ; PVR : population virtuelle réaliste ; RIO Diabetes : rimonabant in obesity and related disorders ; UK GPRD : United Kingdom general practice research database; UKPDS : United Kingdom prospective diabetes study.

\section{Références}

1. http://www.fyma.ucl.ac.be/files/Euler-Bernoulli.pdf 
2. Massol J, Zylberman M, Goehrs JM, et al. Use of the foreign studies: transposition of the results, prediction of the therapeutic effects in the french population, modelling of the public health interest. Therapie 2006; 61: 4819, 491-9

3. Launay O, Hoch D, Saint-Sardos C, et al. Vaccines: Specific features, simulation of impact and medico-economic modelling. Therapie 2010; 65(4): 357-65

4. Bernard GR, Vincent JL, Laterre PF, et al. The Recombinant Human Activated Protein C Worldwide Evaluation in Severe Sepsis (PROWESS) Study Group, Efficacy and safety of recombinant human activated protein $\mathrm{C}$ for severe sepsis. N Engl J Med 2001, 344: 699-709

5. Aegerter P, Auvert B, Buonamico G, et al. Organization and quality control of a clinical database on intensive care medicine in central and suburban Paris. Rev Epidemiol Sante Publique 1998; 46(3): 226-37

6. Riou França L, Launois R, Le Lay K, et al. Cost-effectiveness of drotrecogin alfa (activated) in the treatment of severe sepsis with multiple organ failure. Int J Technol Assess Health Care 2006; 22(1): 101-8

7. Denis P, Lafuma A, Berdeaux G. Medical outcomes of glaucoma therapy from a nation wide representative survey. Clin Drug Investig 2004; 24(6): 343-52

8. Denis P, Lafuma A, Berdeaux G. Medical predictive factors of glaucoma treatment costs. J Glaucoma 2004; 13(4): 283-90

9. Nordmann JP, Lafuma A, Deschaseaux C, et al. Clinical outcomes of glaucoma treatments over a patient lifetime: a Markov model. J Glaucoma 2005; 14(6): 463-9

10. Nordmann JP, Lafuma A, Berdeaux G. Modelling the lifetime economic consequences of glaucoma in France. J Med Econ 2009; 12(1): 9-16

11. Richard JL. The MONICA project. A WHO research project in cardiovascular diseases. Rev Epidemiol Sante Publique 1988; 36(4-5): 325-34

12. Institut National des Statistiques et d'Etudes Economiques. Structure par âge des populations féminine et masculine. http://www.insee.fr/

13. Marchant I, Boissel JP, Kassai B, et al. SCORE should be preferred to Framingham to predict cardiovascular death in French population. Eur J Cardiovasc Prev Rehabil 2009; 16(5): 609-15

14. Marchant I, Nony P, Cucherat M, et al. The global risk approach should be better applied in french hypertensive patients: a comparison between simulation and observation studies. PLOS One 2011; 6: e17508. doi:10.1371/ journal.pone. 0017508

15. Godet-Thobie H, Vernay M, Noukpoape A, et al. Mean blood pressure level and prevalence of hypertension in 18 to 74 year-old adults, ENNS Survey 2006-2007: InVS; 2008. http://www.invs.sante.fr/beh/2008/49_50/ index.htm
16. Boissel JP, Kahoul R, Amsallem E, et al. Towards personalized medicine: exploring the consequences of the effect model-based approach. Personalized Medicine 2011; 8: 581-6. http://www.futuremedicine.com/doi/abs/ 10.2217/pme.11.54

17. Marchant I, Gueyffier F. Towards equitable strategies for hypertension management in cardiovascular prevention. Oral communication, EACPT Budapest 2011

18. Caro JJ. Estimation de l'intérêt de santé publique de rimonabant 20mg dans une population française de patients en surpoids ou obèse et diabétiques de type 2 non contrôlés : L'apport d'une modélisation. ALFEDIAM 2007, Marseille, 23 mars 2007

19. Caro JJ. Pharmacoeconomic analyses using discrete event simulation. Pharmacoeconomics 2005; 23(4): 323-32

20. Cooper K, Brailsford SC, Davies R, et al. A review of health care models for coronary heart disease interventions. Health Care Manag Sci 2006; 9(4): 311-24

21. Caro JJ, Möller J, Getsios D. Discrete event simulation: the preferred technique for health economic evaluations? Value in Health 2010; 13(8): 105660

22. Scheen AJ, Finer N, Hollander P, et al. Efficacy and tolerability of rimonabant in overweight or obese patients with type 2 diabetes: a randomised controlled study. Lancet 2006; 368: 1660-72

23. Boissel JP, Collet JP, Moleur P, et al. Surrogate endpoints, a basis for a rational approach. Eur J Clin Pharmacol 1992; 43: 235-44

24. Gueyffier F, Dib M, Boissel JP. Marqueurs biologiques : biomarqueurs - utilisation au cours du développement et pour l'enregistrement des médicaments. Therapie 2001; 56: 355-61

25. http://www.ispor.org/workpaper/research_practices/PrinciplesofGoodPracticeforDecisionAnalyticModeling-ModelingStudies.pdf

26. http://www.eisbm.org/include/pdf/eisbm_corporatebrochure.pdf

27. Guide to the Methods of NICE Technolgy Appraisals, NICE, 2008. http:// www.nice.org.uk

28. http://www.eisbm.org

29. http://mastersantepublique.univ-lyon1.fr/, spécialité PHAME (PHArmacologie, Modélisation et Essais Cliniques)

Correspondance et offprints : François Gueyffier, Service de Pharmacologie Clinique et Essais Thérapeutiques, Hôpital Louis Pradel, BP Lyon Monchat, 69394 Lyon Cedex 03, France.

E-mail : francois.gueyffier@univ-lyon1.fr 